\title{
Face age and sex modulate the other-race effect in face recognition
}

\author{
Jennifer Wallis • Ottmar V. Lipp • \\ Eric J. Vanman
}

Published online: 30 August 2012

(C) Psychonomic Society, Inc. 2012

\begin{abstract}
Faces convey a variety of socially relevant cues that have been shown to affect recognition, such as age, sex, and race, but few studies have examined the interactive effect of these cues. White participants of two distinct age groups were presented with faces that differed in race, age, and sex in a face recognition paradigm. Replicating the other-race effect, young participants recognized young own-race faces better than young other-race faces. However, recognition performance did not differ across old faces of different races (Experiments 1, 2A). In addition, participants showed an other-age effect, recognizing White young faces better than White old faces. Sex affected recognition performance only when age was not varied (Experiment 2B). Overall, older participants showed a similar recognition pattern (Experiment 3) as young participants, displaying an other-race effect for young, but not old, faces. However, they recognized young and old White faces on a similar level. These findings indicate that face cues interact to affect recognition performance such that age and sex information reliably modulate the effect of race cues. These results extend accounts of face recognition that explain recognition biases (such as the other-race effect) as a function of dichotomous ingroup/outgroup categorization, in that outgroup characteristics are not simply additive but interactively determine recognition performance.
\end{abstract}

J. Wallis · O. V. Lipp • E. J. Vanman

School of Psychology, The University of Queensland,

Brisbane, Australia

J. Wallis $(\square)$

Massachusetts Eye and Ear Infirmary, Department of

Ophthalmology, Harvard Medical School,

243 Charles Street,

Boston, MA 02114, USA

e-mail: jennifer_wallis@meei.harvard.edu
Keywords Face perception $\cdot$ Recognition $\cdot$ Age $\cdot$ Race . Sex $\cdot$ Other-race effect

The face is a source of some of the most important cues for human interaction. As a social species, it is pivotal for humans to analyze person-related information efficiently and, thus, be able to communicate successfully with others (Macrae \& Quadflieg, 2010). For instance, we respond differently depending on whether someone is smiling or looks sad. We might also act differently depending on whether the other person is female or male, young or old, or of the same or a different race than us-all information that can be obtained easily from another person's face. These are mainly conveyed by static (Ekman, 1978) or invariant (Bruce \& Young, 1986) face cues, in contrast to rapid signs or variant expression cues. On the basis of these invariant cues, we may recognize a face that we have seen before by successfully matching visual input with a stored description of the face (e.g., Valentine, 1991). Moreover, the processing of such cues also allows categorization of a face as, for instance, Black, male, and old (Zhao \& Bentin, 2008).

It is important that we differentiate between persons we have met before and those whom we meet for the first time. This is a difficult task, given that we encounter daily a large number of different faces that all share a common set of basic elements. Moreover, the task seems to be complicated further by a set of rather well-established biases. For example, some faces are systematically recognized better than others (Brebner, Krigolson, Handy, Quadflieg, \& Turk, 2011; He, Ebner, \& Johnson, 2011; Meissner \& Brigham, 2001; Wiese, Schweinberger, \& Hansen, 2008; Wright \& Sladden, 2003; Wright \& Stroud, 2002). This recognition bias not only is of theoretical relevance, but also has farreaching practical consequences. For example, White witnesses are more likely to falsely identify an unfamiliar Black 
face as familiar than an unfamiliar White face (Bothwell, Brigham, \& Malpass, 1989; Meissner \& Brigham, 2001), enhancing the likelihood of "false positives" in police lineup situations.

It has been argued that this other-race effect in recognition reflects the manner in which own- and other-race faces are encoded (Levin, 1996, 2000; Sporer, 2001; Valentine, 1991; Young, Bernstein, \& Hugenberg, 2010). Levin (2000) proposed that the dimensions of race (e.g., White and Black) are defined in reference to the outgroup dimension by a feature present/absent mechanism. For example, a White person would code a Black face as Black but a White face as non-Black (and not White). In other words, it is less likely for a White person to notice that someone is also White, whereas he or she probably categorizes a person from a different race according to their race-as Black, for instance. In the latter case, categorical information that defines the ingroup/outgroup differentiation, rather than individuating information, is processed automatically. Similarly, Sporer (2001) suggested that ingroup and outgroup faces are processed differently, enhancing or impairing face recognition, respectively. Face processing is described as a two-step process. First, some visually derived information about a face (e.g., its race, age, or sex) will determine whether it is an ingroup or outgroup face. This evaluation then determines subsequent processing of the face. Perception of an ingroup face leads automatically to the encoding of individuating information. Conversely, when an outgroup face is encountered, perception of the group dimension (e.g., "Black" vs. "White") will induce processing of just this information, making future recognition difficult. Such a process is also incorporated in the sociocognitive framework of face processing proposed by Hugenberg and colleagues (Hugenberg, Young, Bernstein, \& Sacco, 2010; Young, Hugenberg, Bernstein, \& Sacco, 2012), which describes the other-race effect as one instantiation of a broader class of biases. This account argues that whether a perceiver categorizes someone as a member of the ingroup or the outgroup is context dependent and that the emergence of a recognition bias like the other-race effect may be altered by making salient dimensions other than race.

Most research on face recognition has investigated the effects of different facial characteristics separately. Studies on race-face recognition (e.g., Black and White faces) usually use young faces only, whereas studies on age-face recognition (young vs. old faces) generally use White faces (Anastasi \& Rhodes, 2005; Cassia, Picozzi, Kuefner, \& Casati, 2009; Kuefner, Macchi Cassia, Picozzi, \& Bricolo, 2008; Meissner \& Brigham, 2001; Wiese et al., 2008). However, faces usually differ on a number of invariant cues that are processed simultaneously. Only a few studies have looked at the combined effects of invariant facial cues.
Studies measuring event-related potentials have demonstrated that processing of race cues precedes that of sex cues and occurs independently of task demands, suggesting an obligatory process (Ito \& Urland, 2003, 2005). Using a methodology based on the Stroop effect, Montepare and Opeyo (2002) demonstrated that race is judged more quickly and precisely than are age, sex, or emotional expression. These findings suggest that race cues dominate the early categorical processing of faces and that other cues have a lesser effect. However, one might argue that this apparent dominance may reflect obvious luminance differences between faces (i.e., lighter vs. darker skin), whereas evaluation of the other cues (sex, age, expression) might have required an analysis of the faces' spatial characteristics. Hugenberg, Miller, and Claypool (2007) showed that the other-race effect can be overcome with specific instructions (participants were informed about the other-race effect prior to the experiment and then were instructed to individuate outgroup faces), suggesting that observers are able to overcome this default mode of face processing when actively focusing on other facial characteristics.

The present set of experiments investigated the effect of age and sex cues on the other-race effect in face recognition. If, as was discussed previously, race is the dominant invariant cue for face categorization that determines the classification as ingroup or outgroup (Levin, 1996, 2000), one would predict better recognition performance for own-race than for other-race faces, regardless of the other invariant cues present. That is, race is the dominant feature that determines recognition, and the only recognition bias observed will be an other-race effect. Any White faces will be recognized better than any non-White faces by our White participants. Such an outcome would be supportive of frameworks that propose automatic processing of race, such as Levin's (Levin, 1996, 2000). If this framework holds, young and older participants should recognize White faces more accurately than other-race faces, regardless of the faces' age and sex.

Alternatively, the observer may use any of the cues (race, age, or sex) to determine ingroup/outgroup status. In this case, recognition will be more accurate for own-race, ownage, and own-sex faces, as compared with the respective outgroup faces. Thus, various recognition biases will be observed. This outcome would be most supportive of the socio-cognitive approach (Hugenberg et al., 2010; Sporer, 2001), which proposes a dichotomous distinction between ingroup and outgroup, depending on the most relevant cue in a given context. Simply providing faces that differ in more than just their racial characteristics may be sufficient to suggest processing of other cues. If all cues were weighted equally, we would expect participants to recognize faces of their respective ingroup more accurately than faces of any outgroup. Young White female observers, for example, would recognize young White female faces better than any 
other group of faces. Here, we report four experiments investigating recognition performance of faces that differ in race, age, and sex in young, as well as older, participants.

\section{Experiment 1}

The aim of this experiment was to examine whether age and sex cues modulate other-race face recognition, in a group of White, young, female participants. A recognition advantage was hypothesized for faces of an ingroup, as compared with faces of any outgroup. If one of the invariant facial cues served as an outgroup cue, face processing and subsequent recognition should be impaired.

\section{Method}

\section{Participants}

Twenty-two White female first-year psychology students from the University of Queensland, Brisbane, Australia (UQ), 17-27 years of age (mean age: 20.9) participated for course credit.

\section{Materials}

The experimental task was presented using custom-written software run under MS-DOS. Participants sat in a chair approximately $70 \mathrm{~cm}$ from a 17 -in. CRT screen and completed an exposure and a recognition task. Responses were made by pressing one of two buttons on a button box. The buttons were labeled "pleasant" and "unpleasant" for the exposure task and "seen before" and "not seen before" for the recognition task. The position of the labels in each task was counterbalanced across participants. The stimulus materials included 120 grayscale photos, depicting portraits of White, Black, and Asian, young or old women and men, 10 per category. The photos were obtained from the Productive Aging Face Database (Minear \& Park, 2004) and sets of photos created by Dr. Will Hayward and Dr. Jennifer Eberhardt. The remaining photos were drawn from various Web sites. All photos were resized to $260 \times 195$ pixels and presented on a gray background. None of the faces showed facial hair, jewelry, glasses, or any outstanding features, and they all showed a neutral facial expression. The age classification was confirmed in an independent pilot study in which 84 first-year students of the School of Psychology at the University of Queensland (64 female; 1735 years of age, $\mathrm{M}=19.99$; 58 Caucasian, 13 Asian, 1 Aboriginal, 12 other) rated the faces for their perceived age on a scale of $1-9(1=$ young, $9=$ old $)$. Faces were divided into an old and a young group by median split. White and most Black faces were taken from the Productive Aging Face Database that reports each individual's age; thus, we can also report the "real" age for these faces: White young female, 18$27(\mathrm{M}=23)$; White young male, 19-23 ( $\mathrm{M}=21)$; White old female, 41-68 ( $\mathrm{M}=56)$; White old male, 40-68 $(\mathrm{M}=60)$; Black young female, 19-26 ( $\mathrm{M}=22)$; Black young male, 20 $30(\mathrm{M}=24)$; Black old female, 41-78 $(\mathrm{M}=62)$; Black old male, 50-91 $(\mathrm{M}=68)$.

\section{Procedure}

Testing was performed individually in a dedicated laboratory in the presence of the experimenter. Prior to the actual experiment, all participants provided informed consent and information regarding their demographic background. The experiment started with the exposure phase, in which participants were presented with 60 faces centered on the computer screen (see Fig. 1). Faces were evenly divided by race, age, and sex. Two random subsets of 60 photos, 5 per category, were counterbalanced across participants. In the exposure task, faces were presented for $5 \mathrm{~s}$ after a white fixation cross was presented for $500 \mathrm{~ms}$. Participants were asked to rate the faces as pleasant or unpleasant as soon as the face disappeared from the screen (Rhodes, Locke, Ewing, \& Evangelista, 2009; Sporer, 1991). Participants were instructed to be as fast and as accurate as possible with their judgment and were not told about the subsequent assessment of recognition memory. The participant's response triggered the next trial. After the exposure task, participants completed a short filler questionnaire, followed by the recognition task. In the recognition task, the 60 photographs shown before were presented mixed with the remaining faces, resulting in 120 trials. Two random sequences of the 120 photos were used and counterbalanced across participants. Participants were asked to indicate whether they had seen a particular face during the previous task or whether it was a new face. Each face was presented for $5 \mathrm{~s}$. Responses were made after the face had disappeared, and participants were asked to be as fast and accurate as possible with their judgment.

\section{Results and discussion}

To examine the effects of the facial cues race, age, and sex on face recognition performance, an index of discriminability $\left(d^{\prime}\right)$ derived from signal detection theory (Macmillan \& Creelman, 2005) was calculated. All hit and false alarm rates were corrected as recommended by Snodgrass and Corwin (1988), in line with the log linear approach (Stanislaw \& Todorov, 1999).

Preliminary analyses showed no effect of trial sequence or exposure set, and data were collapsed across these factors. Then $d^{\prime}$ scores were subjected to a $3 \times 2 \times 2$ repeated measures ANOVA (using Greenhouse-Geisser corrections to compensate for potential violation of the assumption of sphericity) with race (White, Asian, Black), age (young, old), and sex (female, male) of the faces as within-subjects 
Fig. 1 An overview of the task procedure with example trials. Participants rated faces as pleasant or unpleasant during the exposure phase. After a short intermission, participants indicated whether they had seen a face in the previous phase or whether it was new

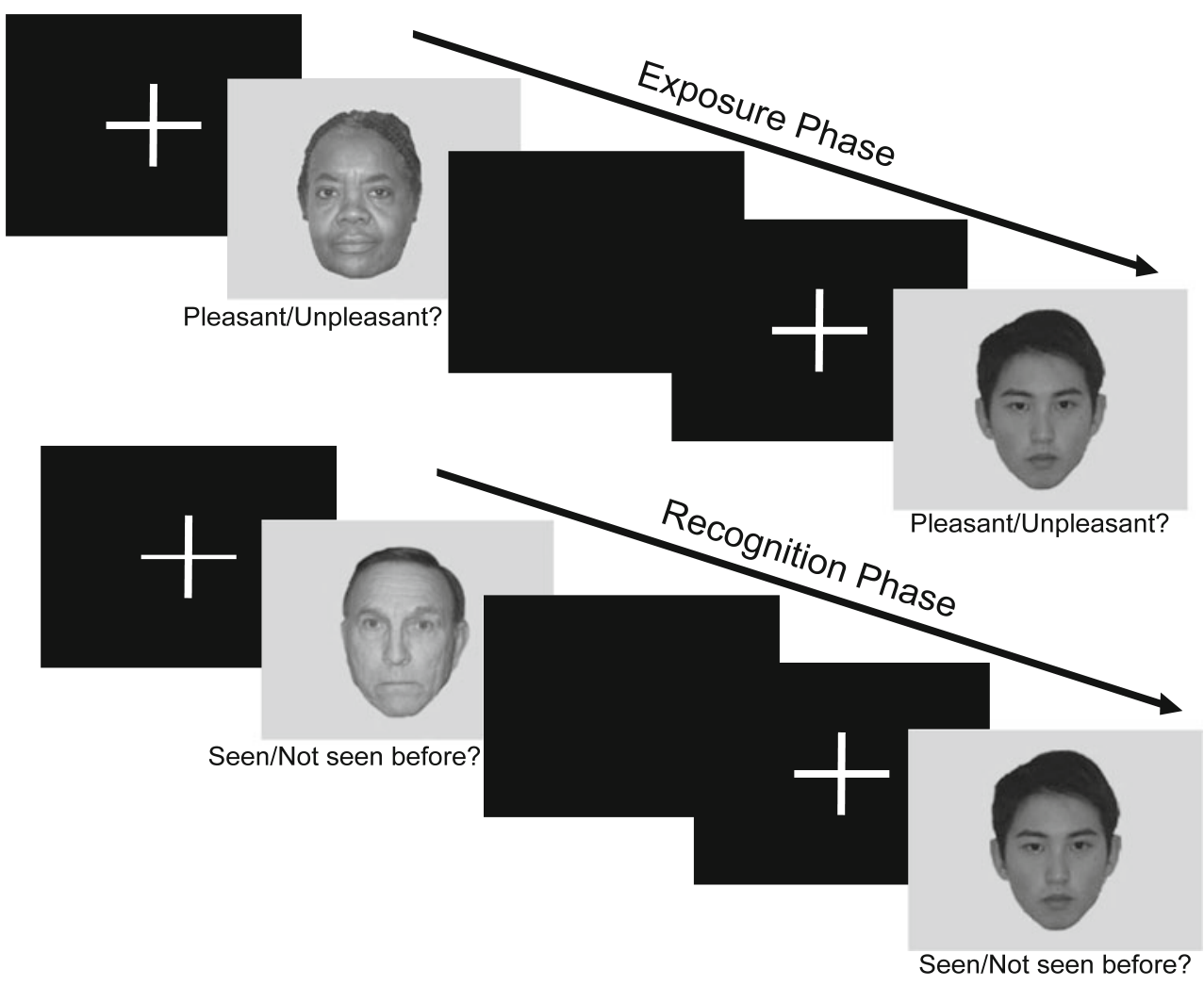

factors. Greenhouse-Geisser epsilon values are reported for main effects and interactions involving repeated measure factors with more than two levels. Follow-up $t$-tests were calculated using the Greenhouse-Geisser corrected error terms and Sidak's correction for multiple comparisons.

Overall, the White, young, female participants showed a significantly higher recognition performance for White faces, as compared with racial outgroup faces. This was illustrated by a significant main effect of race, $F(2,42)=7.67, M S E=$ $0.43, p=.002, \eta_{\mathrm{p}}{ }^{2}=.268, \mathrm{GG} \varepsilon=.919$. As can be seen in Fig. 2, this race effect was apparent only for young faces, as revealed by a race $\times$ age interaction, $F(2,42)=8.97, M S E=$ $0.62, p=.001, \eta_{\mathrm{p}}{ }^{2}=.299, \mathrm{GG} \varepsilon=.934$. Post hoc $t$-tests further clarified these findings: $d^{\prime}$ for young White faces was significantly higher than $d^{\prime}$ for the young Asian and young Black faces, $t(42)=5.07, p<.05$, and $t(42)=3.23, p<.05$, respectively. $d^{\prime}$ did not differ across the old faces from the three racial groups [White/Asian, $t(42)=0.61$; White/Black, $t(42)=1.19$; Asian/Black, $t(42)=0.68 ; p>.05]$. An other-age effect was observed for White faces. Between-age comparisons within race showed the following pattern [White young/old, $t(42)=$ 2.63, $p<.05$; Asian young/old, $t(42)=2.85, p<.05$; Black young/old, $t(42)=1.72, p>.05]$. Although an interaction of race, age, and sex was significant, $F(2,42)=6.64, M S E=$ $0.40, p=.004, \eta_{\mathrm{p}}{ }^{2}=.240, \mathrm{GG} \varepsilon=.899$, sex seemed to have only a modest impact on recognition accuracy. Individual post hoc $t$-tests showed that whereas $d^{\prime}$ was significantly lower for young, female Asian faces than for young, male Asian faces, $t$
$(42)=2.39, p<.05$, there was no difference in $d^{\prime}$ between female and male faces in the remaining race and age categories.

In summary, race affected recognition such that it was better for young racial ingroup faces than for racial outgroup faces. No such difference was evident for old faces, with good recognition performance for all race categories. This

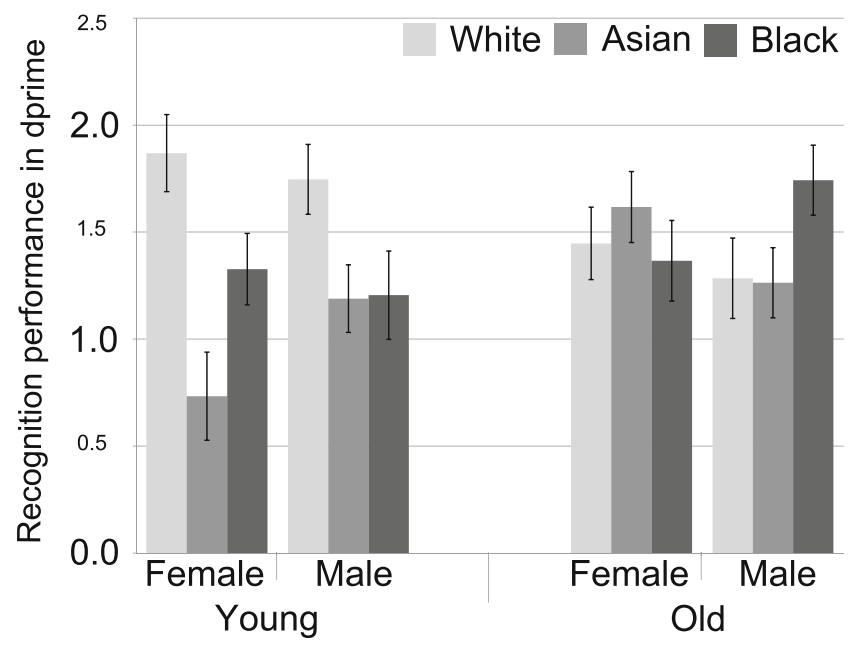

Fig. 2 Recognition performance $\left(d^{\prime}\right)$ for faces that differed in race, age, and sex in Experiment 1 (error bars represent standard errors of the means). Participants showed better recognition for young own-race than for young other-race faces. No difference in recognition for old faces was found across races 
finding was unexpected and is inconsistent with previous literature on the other-race effect. One possible explanation, however, is that this pattern of findings was due to the particular photo set used in Experiment 1. To address this issue, we replicated Experiment 1 with a different set of faces (Experiment 2A) and in a different design (Experiment $2 \mathrm{~B})$, using the age cue as a between-subjects factor.

\section{Experiment 2A}

This experiment replicated Experiment 1 using a different photo set. To increase generalizability, colored photos were used. On the basis of Experiment 1, we expected better recognition for own-race faces (White) than other-race faces (Chinese), for young, but not old, faces.

\section{Method}

\section{Participants and materials}

Nineteen White female students from UQ, 17-30 years of age (mean age: 19.7), participated for course credit. Materials were the same as in Experiment 1, except that faces were chosen from a new set of 80 colored portraits of White and Chinese, young and old, women and men. White faces were obtained from the Productive Aging Face Database (Minear \& Park, 2004); Chinese faces were drawn from various public Web sites. Ages for the White faces were the following: White young female, $18-25, M=21$; White young male, $18-23, M=21$; White old female, 56$69, M=64$; White old male, 55-69, $M=64$.

\section{Procedure}

The procedure in Experiment 2A was the same as that in Experiment 1, with the following exceptions. Face presentation duration was reduced to $2 \mathrm{~s}$ during exposure and recognition. Forty faces were presented in the exposure phase. In the recognition phase, the 40 faces shown before were combined with the same number of new, unseen faces, resulting in 80 trials.

Results and discussion

The discriminability index $d^{\prime}$ was subjected to a $2 \times 2 \times 2$ repeated measures ANOVA (race [White, Chinese] $\times$ age [young, old] $\times$ sex [female, male]. Across stimulus groups, $d^{\prime}$ scores differed, replicating the findings from Experiment 1. Participants were significantly better at recognizing White than Chinese faces [main effect of race, $F(1,18)=15.83$, $\left.M S E=0.27, p=.001, \eta_{\mathrm{p}}{ }^{2}=.468\right]$. A race $\times$ age interaction, $F(1,18)=4.17, M S E=0.17, p=.056, \eta_{\mathrm{p}}{ }^{2}=.188$, however, revealed that this race effect tended to be present only for the young faces (see Fig. 3). Whereas the mean $d^{\prime}$ value for young White faces was significantly higher than that for young Chinese faces, $t(18)=4.96, p<.05$, no such difference was found for the old faces, $t(18)=2.07, p>.05$.

In summary, an other-race effect was found for young faces, thus replicating the findings from Experiment 1, indicating that they are not specific to the stimulus set used or the participant group tested. Both experiments indicate that age modulates the recognition of faces from different racial groups. If these different recognition patterns are typical for the respective age group, they should occur regardless of whether the stimulus faces presented in a particular experiment vary in age. In other words, the race effect should emerge among young faces, but not among old faces of different race, even if faces of only one age group are presented to each participant. Experiment 2B tested this hypothesis by presenting participants with either young or old faces.

\section{Experiment 2B}

Method

\section{Participants and materials}

Sixteen White female students from UQ, 18-36 years of age (mean age: 24.8), participated for course credit or a payment of 10 AUD. Materials were the same as those in Experiment 1 (grayscale photos), except that the Black faces were not

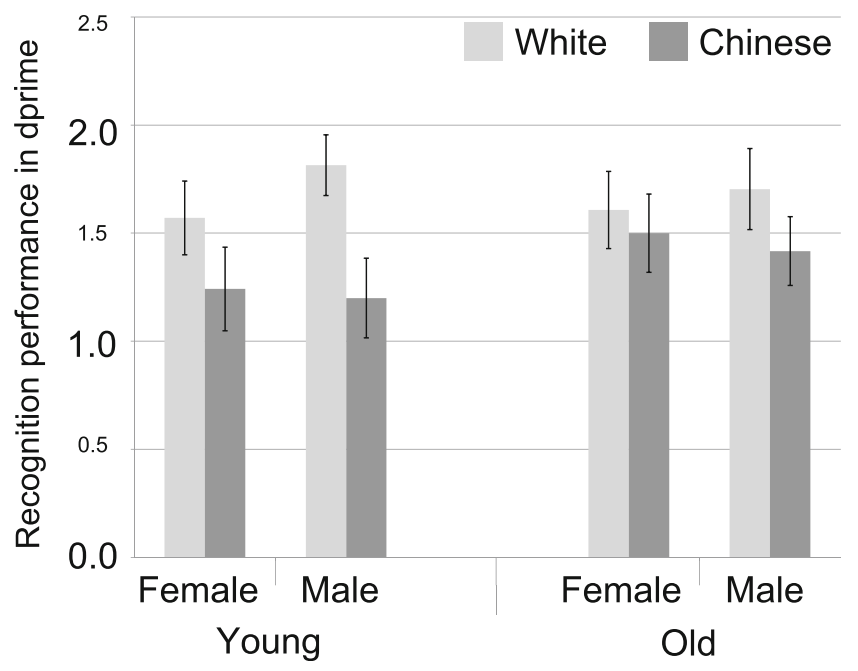

Fig. 3 Recognition performance $\left(d^{\prime}\right)$ for faces that differ in race, age, and sex in Experiment 2A (error bars represent standard errors of the means). Participants showed better recognition for young White faces than for young Chinese faces but showed no difference in recognition for old White and old Chinese faces 
used. Half of the participants saw only young faces, and the others only old faces.

\section{Procedure}

The procedure in Experiment $2 \mathrm{~B}$ was the same as that in Experiment 1, with the following exceptions. Participants would see either old or young faces, evenly divided by sex (female, male) and race (Chinese, White). Twenty faces were presented in the exposure phase for $1 \mathrm{~s}$ each. In the recognition phase, the 20 photographs shown before were combined with the same number of new, unseen faces, of the same age group as those used during exposure. Each face was presented for $1 \mathrm{~s}$.

Results and discussion

A $2 \times 2 \times 2$ mixed measures ANOVA assessed recognition performance for young versus old faces, with race (White, Chinese) and sex (female/male) of the faces as withinsubjects factors and age (young/old) as the betweensubjects factor. The ANOVA revealed a significant main effect of sex, $F(1,14)=6.84, M S E=0.41, p=.020, \eta_{\mathrm{p}}{ }^{2}=$ .328 , demonstrating better recognition performance for female than for male faces. A main effect of race, $F(1,14)=$ 10.20, MSE $=0.58, p=.006, \eta_{\mathrm{p}}{ }^{2}=.422$, illustrated better recognition performance for White faces than for Chinese faces. As is shown in Fig. 4, this race effect was evident only for young faces [race $\times$ age interaction, $F(1,14)=8.42$, $\left.M S E=0.58, p=.012, \eta_{\mathrm{p}}{ }^{2}=.376\right]$. Post hoc $t$-tests showed that $d^{\prime}$ was higher for young White faces than for young

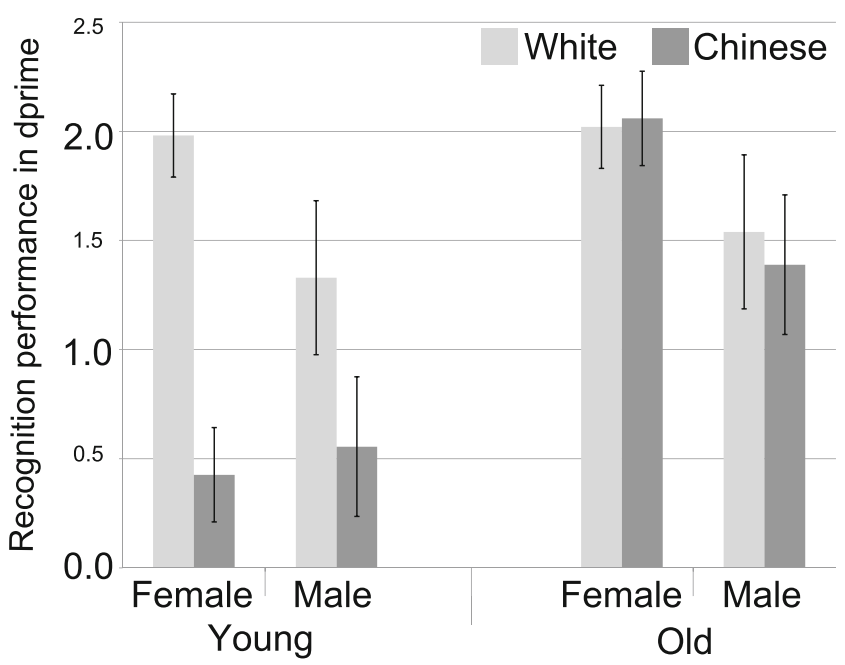

Fig. 4 Recognition performance $\left(d^{\prime}\right)$ for faces that differ in race, age, and sex in Experiment 2B (error bars represent standard errors of the means). Participants who were presented with young faces showed better recognition for White than for Chinese faces. However, participants who saw old faces showed no difference in recognition for White and Chinese faces
Chinese faces, $t(14)=6.09, p<.05$, whereas no difference was found for the old faces, $t(14)=0.29, p>.05$.

In summary, these results support the findings of Experiment 1 (and 2A) demonstrating an other-race effect only for recognition of young faces, but not for old faces. Even when age differences within the face set were eliminated, recognition for faces of various racial groups seemed to be dependent on the respective ages of the faces. Moreover, it seems that by reducing the number of invariant face cues varied, sex of the face became more salientfurther indication that the various cues seem to interactively affect face recognition. Female participants were significantly better at recognizing female faces than male faces, suggesting that sex cues were processed in other-race faces, as well as own-race faces (see also Zhao \& Hayward, 2010).

\section{Experiment 3}

In this experiment, recognition performance of older participants was investigated. Experiments 1, 2A, and 2B showed that in younger participants, the other-race effect was found for young, but not for old, faces. Older individuals, however, may show an other-race effect when asked to recognize their age peers. We hypothesized that older adults show an other-race effect for old faces and an other-age effect with better recognition for old than for young faces within their own race (White). That is, we expected a recognition advantage for faces of an ingroup, as compared with faces of any outgroup; such classification will depend on participants' age. Alternatively, old age may change faces in a way that the other-race effect can be overcome (see the discussion on the caricature effect below; e.g., Rodriguez, Bortfeld, Rudomin, Hernandez, \& Gutierrez-Osuna, 2008).

Method

\section{Participants}

Seventeen White participants (4 males; mean age, 66.8; range, 52-83) were recruited at the vision rehabilitation clinic at the Massachusetts Eye and Ear Infirmary, Boston. One person was not willing to provide an exact age but reported it as 60 plus. Participants were either family members of clinic patients or staff of the hospital. All participants had normal or corrected-to-normal vision.

\section{Materials}

Materials were the same as those in Experiment 1, except for the following changes. The task was presented using the Psychtoolbox Library under MATLAB on a Macintosh 
laptop computer (MacbookPro, 13-in. screen). Participants pressed the "M" and "C" keyboard keys for responses.

\section{Procedure}

The procedure was the same as that in Experiment 1.

Results and discussion

\section{Sensitivity (d')}

The $d^{\prime}$ values were calculated as described in Experiment 1 . However, face sex was coded as same/different from each participant's sex, so as to allow inclusion of the 4 male observers. ${ }^{1}$ As can be seen in Fig. 5, overall, old participants appear to show a recognition pattern similar to that of the young participants. The main difference between young and old participants seems to be that old observers showed relatively accurate recognition for all White faces.

While old participants showed an other-age effect consistent with our hypothesis that they would recognize old faces more accurately than young faces [main effect of age, $F(1,16)$ $\left.=10.04, M S E=0.46, p=.006, \eta_{\mathrm{p}}{ }^{2}=.386\right]$, this effect was mainly driven by the other-race faces [race $\times$ age interaction, $F$ $\left.(2,32)=7.13, M S E=0.56, p=.004, \eta_{\mathrm{p}}{ }^{2}=.308, \mathrm{GG} \varepsilon=.923\right]$. In fact, old participants showed no difference in recognition for young and old White faces, $t(32)=1.03, p>.05$. However, they recognized old Asian faces more accurately than young Asian faces; the difference in recognition between young and old Black faces did not reach significance [Asian, $t(32)=4.08$, $p<.05$; Black, $t(32)=1.88, p>.05]$.

An other-race effect was also observed with more accurate recognition for White than for Asian and Black faces [main effect of race, $F(2,32)=13.14, M S E=0.38, p<.001$, $\left.\eta_{\mathrm{p}}{ }^{2}=.451, \mathrm{GG} \varepsilon=.782\right]$. This is mainly driven by recognition of young faces.

\section{Response bias (c)}

An overview of response bias $(c)$ for performance observed in this experiment, as well as for the young participants' performance in Experiment 1, is presented in Fig. 6. Old and young participants show a similar pattern of response bias [the only differential effect is a subject age $\times$ sex interaction, $F(1,37)=4.33, M S E=0.11, p=.044, \eta_{\mathrm{p}}{ }^{2}=.105$, showing that young participants are more likely to identify other-sex faces than own-sex faces as "not seen before," $t(37)=3.47$, $\mathrm{p}<.05$, whereas old participants show no such difference, $t$ $(37)=0.55, p>.05]$. In general, old and young participants

\footnotetext{
${ }^{1}$ The key pattern of results does not change when the male observers are excluded from the analysis.
}

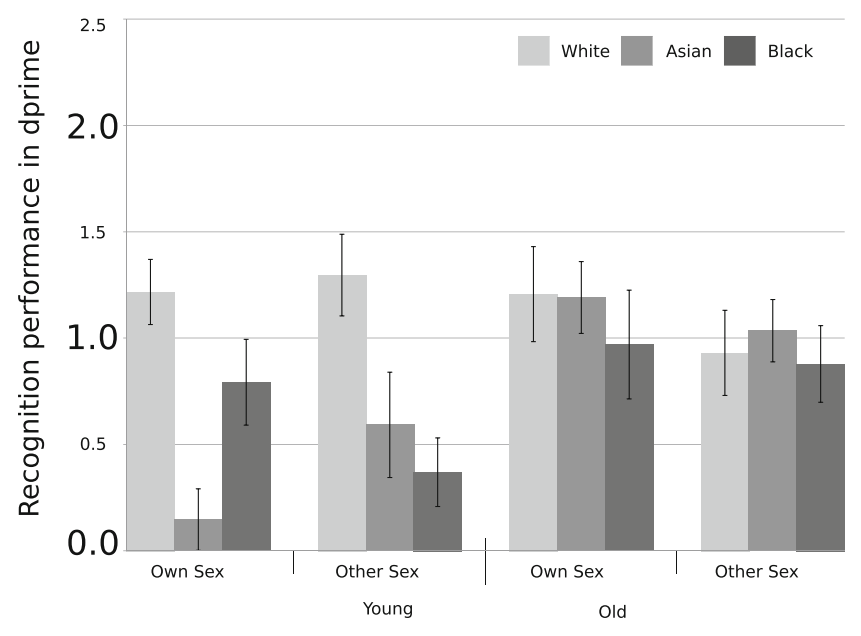

Fig. 5 Recognition performance $\left(d^{\prime}\right)$ for faces that differ in race, age, and sex in older adults, Experiment 3 (error bars represent standard errors of the means). Older participants show better recognition for young own-race faces than for young other-race faces. They recognize all White faces relatively well

seem to be relatively more likely to identify young otherrace faces as previously seen (i.e., liberal bias), as compared with the other groups of faces [race $\times$ age interaction, $F(2$, $\left.74)=12.71, M S E=0.25, p<.001, \eta_{\mathrm{p}}{ }^{2}=.256, \mathrm{GG} \varepsilon=.872\right]$.

In summary, we observe some support for recognition biases dependent on participants' race and age. In contrast to the young participants, first, old participants showed no other-age effect within White faces. That is, whereas young participants recognized White faces of their own age group better than old White faces, old participants showed no difference between White young and old faces. Second, old participants showed generally lower $d^{\prime}$ values than did the young participants. Surprisingly, the recognition pattern was otherwise comparable to the young observers' performance in Experiment 1, suggesting that old age may change faces of various races in such a way that the other-race effect can be overcome. Research on face caricatures may offer an explanation for this finding. Caricatures of faces are more memorable than are the veridical faces but also appear older (O'Toole, Vetter, Volz, \& Salter, 1997). Thus, older faces may be easier to recognize, since certain parts become more pronounced when aging.

\section{General discussion}

Four experiments examined the extent to which race, age, and sex affect face recognition. In line with the sociocognitive approach, we hypothesized that faces of an ingroup would be recognized more accurately than faces of an outgroup (Hugenberg et al., 2010). Across the first three experiments, young participants' recognition performance was tested. An other-race effect was found only for 
Fig. 6 Overview of bias $(c)$ for Experiments 1 (young participants) and 3 (old participants). Own-race face categories are marked with a gray square background

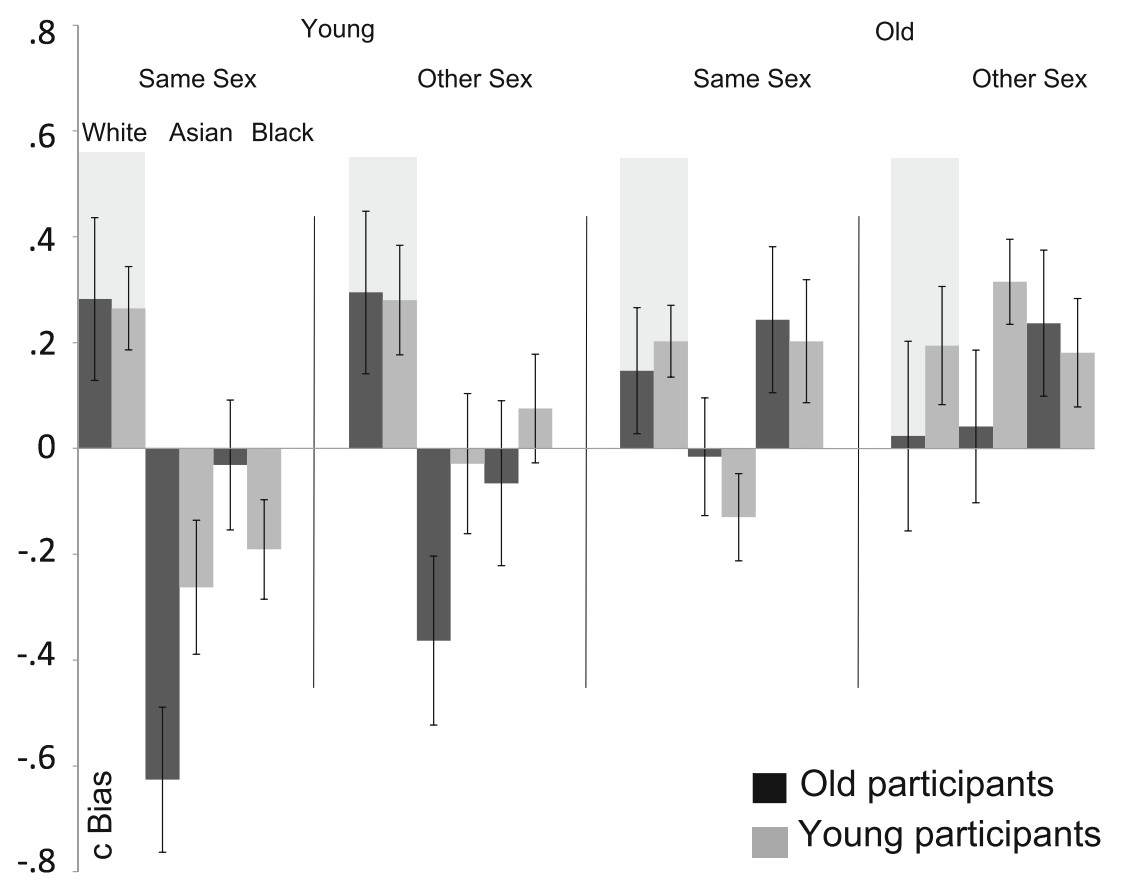

recognition of young faces. No difference in recognition was found across old faces of different races (Experiments 1 and 2A). That is, race differences determined processing and subsequent recognition such that young own-race faces were recognized better than young other-race faces. However, all old faces were recognized at a similar high level, irrespective of their race and sex. Sex of the face had a differential impact on recognition performance only when the faces varied in race and sex (Experiment 2B), but not when age was varied as well. That is, when either only young or old faces of different race and sex were presented, female participants were better at recognizing female faces than male faces.

Older participants showed a pattern of face recognition (Experiment 3) similar to that of the young observers. Surprisingly, old participants also showed an other-race effect for young faces, but not old faces. In addition, and in line with previous reports (He et al., 2011; Wiese et al., 2008; Wolff, Wiese, \& Schweinberger, 2012), older adults showed relatively good recognition performance for both their ingroup (White, old faces) and young White faces (i.e., they did not show an other-age effect). This suggests that older adults may not categorize young adults of their own race as members of an outgroup. It is reasonable to assume that older adults equally see young adults of their own race, as well as their peers, as ingroup. Alternatively, they may have acquired sufficient experience in processing younger faces throughout their life. However, this interpretation should be taken with caution, since the literature on the other-age effect is relatively inconsistent, perhaps due to varying paradigms or age groups used, and there are reports of other-age effects in older participants (e.g., Anastasi \& Rhodes, 2005; Wright \& Stroud, 2002).
One might argue that our findings reflect on the particular face set employed in our studies, since young and old participants, when tested with the same set of faces, displayed similar results (Experiments 1 and 3). However, we replicated the results in Experiment 1 using a different face set presented in color (Experiment 2A). Moreover, Wiese (2012) recently reported the same recognition pattern in young participants; that is, young participants recognized young White faces better than White old faces and showed an other-race effect exclusively for young faces. This additional report, using a different face set and participant sample, supports the generality of the findings described above.

The present results have both methodological and theoretical implications for the study of face recognition. Whereas the other-race effect based on observer and poser race differences is well investigated, little is known about how simultaneous perception of age and sex cues affects subsequent recognition of own- and other-race faces. We showed that the other-race effect might be limited to young faces, the faces usually employed in research on recognition of other-race faces. If faces differ only in one cue (e.g., race), however, further face processing would most likely be based on this particular cue and its variations. Thus, in a typical face recognition study investigating the other-race effect, racial differences are the most salient cue, possibly facilitating the occurrence of an other-race effect. To the best of our knowledge, this is the first study investigating the effect of multiple facial cues on recognition in older adults, and further research is required.

The present findings suggest that various face cues interact to determine face recognition. Old age, in particular, seems to influence recognition of own- and other-race faces 
in a way that the other-race effect can be overcome. Findings from face caricature research may provide an explanation for the relatively good recognition performance for older faces across races for both old and young participants. Caricatures of faces are more memorable than the veridical faces (this may be true for unfamiliar faces only; see Kaufmann \& Schweinberger, 2008) but are also judged as appearing older (O'Toole et al., 1997). Byatt and Rhodes (1998) asked participants to study faces and then to recognize caricatured versions of these faces. Original faces were caricatured along either a correct norm (i.e., distorted relative to the average of the same race as the target) or an incorrect norm (i.e., distorted relative to the average of a different race). Recognition was better for right-norm caricatures, irrespective of race. The aging process may render faces more distinct, as does a right-norm caricature, leading to equally good recognition performance of older faces across different races. Older faces may become more recognizable, since certain parts become more pronounced when people age (Bartlett, Grossman, \& Whitaker, 1991; Behrents, 1985). Rodriguez, Bortfeld, Rudomin, Hernandez, and Gutierrez-Osuna (2008) showed that exposure to other-race caricatures facilitated recognition for other-race faces. Participants who were familiarized with caricatures of different races exhibited no other-race effect in a subsequent recognition test on the veridical faces. The authors suggested that the other-race caricatures guided perceivers' attention to relevant features that are usually neglected.

The present findings are broadly consistent with accounts that propose ingroup/outgroup dependent recognition performance, such as the socio-cognitive framework, but suggest some qualifications. Ingroup/outgroup classification is argued to depend on the most relevant cue in a given context. Providing faces that differ on more than just their racial characteristics seems to be sufficient to suggest processing of other cues. When race, age, and sex information were available, race and age were processed in preference to sex. This observation leads to two proposals. First, outgroup cues do not have an "all or nothing" effect for face processing. Sporer's (2001) ingroup/outgroup model of face processing predicts that once an outgroup feature is detected, further face processing will be impaired, resulting in impaired recognition. Levin $(1996,2000)$ argued that race is processed automatically in other-race faces. As a result, more categorical information is processed for these faces, and thus they are recognized comparatively poorly. We argue that, instead, the detection of other-race cues seems not to impair further processing of other invariant face cues (such as age). The presence of some outgroup cues-for example, age cues, in the present case - can ameliorate the effects of other outgroup cues, such as race. Participants recognized old other-race faces better than young otherrace faces. Second, our findings show that facial cues are not processed independently and then simply combined, such that members of an outgroup defined as such by two cues would be recognized less well than members of an outgroup defined as such by just one cue. Whereas participants recognized most groups less well than their ingroup, there was no consistent increase in recognition from a "triple" outgroup (e.g., Black old males for our young white, female participants) to a "double" outgroup (Black old female for young white, female participants) and to a "simple" outgroup to the ingroup.

When studying face recognition, it is important to consider that numerous face cues are available simultaneously. Previous research has shown that a recognition bias, with better recognition for the ingroup, results when a clear differentiation into ingroup and outgroup along one dimension is possible. Our findings suggest that the perception of age and sex cues can modulate the effects of race cues and that these cues interactively influence face processing and recognition.

Acknowledgements This research was supported under the Australian Research Council's Discovery Projects funding scheme (project number DP110100460) and by an UQRS/UQIRTA fellowship to Jennifer Wallis.

We would like to thank Paul Jackson, who programmed the tasks for Experiments 1, 2A, and 2B, Dr. Will Hayward and Dr. Jennifer L. Eberhardt for permission to use their photos, Thomas Wallis for his feedback on earlier versions of the manuscript, as well as programming the task for Experiment 3, and the staff at the vision rehabilitation clinic who facilitated participant recruitment for Experiment 3.

\section{References}

Anastasi, J. S., \& Rhodes, M. G. (2005). An own-age bias in face recognition for children and older adults. Psychonomic Bulletin \& Review, 12(6), 1043-1047.

Bartlett, S. P., Grossman, R., \& Whitaker, L. A. (1991). Age-related Changes of the Craniofacial Skeleton: An Anthropometric and Histologic Analysis. Journal of the American Society of Plastic Surgeons, 90, 585-591.

Behrents, R. G. (1985). Growth in the aging craniofacial skeleton. Paper presented at the Center for Human Growth and Development, The University of Michigan, Ann Arbor, MI.

Bothwell, R. K., Brigham, J. C., \& Malpass, R. S. (1989). Cross-Racial Identification. Personality and Social Psychology Bulletin, 15, $19-25$.

Brebner, J. L., Krigolson, O., Handy, T. C., Quadflieg, S., \& Turk, D. J. (2011). The importance of skin color and facial structure in perceiving and remembering others: an electrophysiological study. Brain Research, 1388, 123-133. doi:10.1016/j.brainres.2011.02.090

Bruce, V., \& Young, A. (1986). Understanding face recognition. British Journal of Psychology, 77(Pt 3), 305-327.

Byatt, G., \& Rhodes, G. (1998). Recognition of own-race and otherrace caricatures: implications for models of face recognition. Vision Research, 38(15-16), 2455-2468.

Cassia, V. M., Picozzi, M., Kuefner, D., \& Casati, M. (2009). Why mix-ups don't happen in the nursery: evidence for an experiencebased interpretation of the other-age effect. Quarterly Journal of 
Experimental Psychology, 62(6), 1099-1107. doi:10.1080/ 17470210802617654

Ekman, P. (1978). Facial signs: Facts, fantasies, and possibilities. In T. Sebeok (Ed.), Sight, sound, and sense (pp. 124-156). Bloomington, IN: Indiana University Press.

He, Y., Ebner, N. C., \& Johnson, M. K. (2011). What Predicts the Own-Age Bias in Face Recognition Memory? Social Cognition, 29(1), 97-109.

Hugenberg, K., Miller, J., \& Claypool, H. M. (2007). Categorization and individuation in the cross-race recognition deficit: Toward a solution to an insidious problem. Journal of Experimental Social Psychology, 43, 334-340.

Hugenberg, K., Young, S. G., Bernstein, M. J., \& Sacco, D. F. (2010). The categorization-individuation model: an integrative account of the other-race recognition deficit. Psychological Review, 117(4), 1168-1187. doi:10.1037/a0020463

Ito, T. A., \& Urland, G. R. (2003). Race and gender on the brain: electrocortical measures of attention to the race and gender of multiply categorizable individuals. Journal of Personality and Social Psychology, 85(4), 616-626. doi:10.1037/0022-3514.85.4.616

Ito, T. A., \& Urland, G. R. (2005). The influence of processing objectives on the perception of faces: an ERP study of race and gender perception. Cognitive, Affective \& Behavioral Neuroscience, 5(1), 21-36.

Kaufmann, J. M., \& Schweinberger, S. R. (2008). Distortions in the brain? ERP effects of caricaturing familiar and unfamiliar faces. Brain Research, 1228, 177-188. doi:10.1016/j.brainres.2008.06.092

Kuefner, D., Macchi Cassia, V., Picozzi, M., \& Bricolo, E. (2008). Do all kids look alike? Evidence for an other-age effect in adults. Journal of Experimental Psychology: Human Perception and Performance, 34(4), 811-817. doi:10.1037/0096-1523.34.4.811

Levin, D. T. (1996). Classifying faces by race: The structure of face categories. Journal of Experimental Psychology. Learning, Memory and Cognition, 22, 1364-1383.

Levin, D. T. (2000). Race as a visual feature: using visual search and perceptual discrimination tasks to understand face categories and the cross-race recognition deficit. Journal of Experimental Psychology: General, 129(4), 559-574.

Macmillan, N. A., \& Creelman, C. D. (2005). Detection Theory: A User's Guide (2nd ed.). Mahwah, N.J: Lawrence Erlbaum Associates.

Macrae, C. N., \& Quadflieg, S. (2010). Person perception. In S. Fiske, D. T. Gilbert, \& G. Lindzey (Eds.), The handbook of Social Psychology (5th ed., pp. 428-463). New York: McGraw-Hill.

Meissner, C. A., \& Brigham, J. C. (2001). Thirty years of investigating the own-race bias in memory for faces: A meta-analytic review. Psychology, Public Policy, and Law, 7(1), 3-35. doi:10.1037// 1076-8971.7.1.3

Minear, M., \& Park, D. C. (2004). A lifespan database of adult facial stimuli. Behavior Research Methods, 36(4), 630-633.

Montepare, J. M., \& Opeyo, A. (2002). The Realtive Salience of Physiognomic Cues in Differential Faces: A Methodological Tool. Journal of Nonverbal Behavior, 26, 43-59.

O'Toole, A. J., Vetter, T., Volz, H., \& Salter, E. M. (1997). Threedimensional caricatures of human heads: distinctiveness and the perception of facial age. Perception, 26(6), 719-732.
Rhodes, G., Locke, V., Ewing, L., \& Evangelista, E. (2009). Race coding and the other-race effect in face recognition. Perception, $38(2), 232-241$.

Rodriguez, J., Bortfeld, H., Rudomin, I., Hernandez, B., \& GutierrezOsuna, R. (2008). The reverse-caricature effect revisited: Familiarization with frontal facial caricatures improves veridical face recognition. Applied Cognitive Psychology, 23, 733-742.

Snodgrass, J. G., \& Corwin, J. (1988). Pragmatics of measuring recognition memory: applications to dementia and amnesia. Journal of Experimental Psychology: General, 117(1), 34-50.

Sporer, S. L. (1991). Deep-deeper-deepest? Encoding strategies and the recognition of human faces. Journal of Experimental Psychology: Learning, Memory \& Cognition, 17(2), 323-333.

Sporer, S. L. (2001). Recognizing faces of other ethnic groups: An integration of theories. Psychology, Public Policy, and Law, 7(1), 36-97. doi:10.1037//1076-8971.7.1.36

Stanislaw, H., \& Todorov, N. (1999). Calculation of signal detection theory measures. Behavior Research Methods, Instruments \& Computers, 31(1), 137-149.

Valentine, T. (1991). A unified account of the effects of distinctiveness, inversion, and race in face recognition. Quarterly Journal of Experimental Psychology A, 43(2), 161-204.

Wiese, H. (2012). The role of age and ethnic group in face recognition memory: ERP evidence from a combined own-age and own-race bias study. Biological Psychology, 89(1), 137-147. doi:10.1016/ j.biopsycho.2011.10.002

Wiese, H., Schweinberger, S. R., \& Hansen, K. (2008). The age of the beholder: ERP evidence of an own-age bias in face memory. Neuropsychologia, 46(12), 2973-2985. doi:10.1016/ j.neuropsychologia.2008.06.007

Wolff, N., Wiese, H., \& Schweinberger, S. R. (2012). Face Recognition Memory Across the Adult Life Span: Event-Related Potential Evidence From the Own-Age Bias. Psychology and Aging, Jun 25 , No Pagination Specified. doi: 10.1037/ a0029112

Wright, D. B., \& Sladden, B. (2003). An own gender bias and the importance of hair in face recognition. Acta Psychologica, 114(1), 101-114.

Wright, D. B., \& Stroud, J. N. (2002). Age differences in lineup identification accuracy: people are better with their own age. Law and Human Behavior, 26(6), 641-654.

Young, S. G., Bernstein, M. J., \& Hugenberg, K. (2010). When Do Own-Group Biases In Face Recognition Occur? Encoding Versus Post-Encoding. Social Cognition, 28(2), 240-250.

Young, S. G., Hugenberg, K., Bernstein, M. J., \& Sacco, D. F. (2012). Perception and motivation in face recognition: a critical review of theories of the cross-race effect. Personality and Social Psychology Review, 16(2), 116-142. doi:10.1177/ 1088868311418987

Zhao, L., \& Bentin, S. (2008). Own- and other-race categorization of faces by race, gender, and age. Psychonomic Bulletin \& Review, 15(6), 1093-1099. doi:10.3758/PBR.15.6.1093

Zhao, M. T., \& Hayward, M. G. (2010). Holistic processing underlies gender judgments of faces. Attention, Perception, \& Psychophysics, 72(3), 591-596. 